\title{
DETERMINAÇÃo CONDUTOMÉTRIA DE CARBONATO RESIDUAL DO CALCÁRIO APLICADO NO SOLO POR ANÁLISE EM FLUXO
}

Lilka Isuzu Kawazaki, Mario Miyazawa, Marcos Antonio Pavan e Julio Cezar Franchini

Instituto Agronômico do Paraná - IAPAR - CP 481 - 86001-970 - Londrina - PR

Recebido em 30/8/99; aceito em 21/12/99

\begin{abstract}
CONDUCTIVIMETRIC DETERMINATION OF RESIDUAL CARBONATE FROM LIME AMENDED SOIL USING FLOW ANALYSIS. The objective of this study was to evaluate a flow injection system for determination of residual $\mathrm{CO}_{3}{ }^{2-}$ in soil amended with lime material. It was used a closed system were the $\mathrm{CO}_{2}$ released from soil sample acidified with $0.5 \mathrm{~mol} \mathrm{~L}^{-1} \mathrm{HCl}$ was capted in a 0.2 mol $\mathrm{L}^{-1} \mathrm{NaOH}$ solution. After $16 \mathrm{~h}$ the capted $\mathrm{CO}_{2}$ was determined by conductivimetry using a flow injection system. The results obtained by the proposed method were significantly correlated with those reported in soil samples used by the International Soil Analytical Exchange Programe. The regression equation was: $y=0.987 x-0.075 r=0.996, P>0.01$. For acid soils amended with $\mathrm{CaCO}_{3}$ the method showed a deviation error of $2.7 \%$, detection limit was $0.077 \mathrm{mmol} \mathrm{kg}^{-1}$ of $\mathrm{CO}_{3}^{2-}$, and a recovery of $99.7 \%$ of the total $\mathrm{CO}_{3}{ }^{2-}$ added in soil sample. The method was easily adapted for routine determination of residual $\mathrm{CO}_{3}{ }^{2-}$ in soil samples with an analytical frequency of $\mathbf{4 0}$ samples per hour.
\end{abstract}

Keywords: lime; soil analysis; FIA system.

\section{INTRODUÇÃO}

A maioria dos solos brasileiros são ácidos e apresentam níveis tóxicos de alumínio e baixo teor de cálcio, características que reduzem a produtividade agrícola. A correção do solo com calcário é a prática recomendada para elevar o $\mathrm{pH}$, neutralizar o Al tóxico e fornecer $\mathrm{Ca}$ e $\mathrm{Mg}$ para as plantas.

Os materiais corretivos mais utilizados na neutralização da acidez dos solos são os calcários dolomítico $\left(\mathrm{CaCO}_{3}+\right.$ $\left.\mathrm{MgCO}_{3}\right)$ e calcítico $\left(\mathrm{CaCO}_{3}\right)$. A cinética da reação do calcário depende do grau de acidez do solo, da granulometria e da intimidade da mistura com o solo. Frequentemente, as quantidades de $\mathrm{H}^{+}$e $\mathrm{Al}^{3+}$ neutralizadas pelo $\mathrm{CaCO}_{3}$ adicionado, são menores do que a esperada, principalmente para doses elevadas ${ }^{1}$. Como possíveis causas deste comportamento, atribuiu-se à dissolução incompleta do $\mathrm{CaCO}_{3}$, às perdas de $\mathrm{Ca}$ por lixiviação e às reações de coprecipitação de $\mathrm{Ca}$ com hidróxidos de $\mathrm{Al}$ e de $\mathrm{Fe}^{1}$. A determinação de $\mathrm{Ca}$ trocável e $\mathrm{CO}_{3}{ }^{2-}$, possibilitará o cálculo exato do balanço da reação do $\mathrm{CaCO}_{3}$ aplicado em solos ácidos.

$\mathrm{O}$ método de determinação gravimétrica de $\mathrm{CO}_{3}{ }^{2-}$ descrito por Allison e Moodie ${ }^{2}$ baseia-se na perda de massa de $\mathrm{CO}_{2}$ do solo pela adição do $\mathrm{HCl}$ em excesso. A maior desvantagem do método é a sua aplicação somente para solos que contêm mais de $200 \mathrm{mmol}_{\mathrm{c}} \mathrm{kg}^{-1}$ de $\mathrm{CO}_{3}{ }^{2-}$. Ashworth ${ }^{3}$ desenvolveu um método potenciométrico para análise de $\mathrm{CO}_{3}{ }^{2-}$ de solo calcário em rotina. $\mathrm{O}$ princípio deste método foi o aumento de $\mathrm{pH}$ da solução de ácido acético pelo $\mathrm{CO}_{3}{ }^{2-}$. A sensibilidade deste método foi semelhante ao anterior, insuficiente para avaliar $\mathrm{CO}_{3}{ }^{2-}$ residual do calcário aplicado em solos ácidos, onde as concentrações estão entre 1 a $10 \mathrm{mmol}_{\mathrm{c}} \mathrm{kg}^{-1}$. Por isso é necessário um método mais sensível para o estudo da reação do calcário aplicado em solo ácido.

Jenkinson e Powlson ${ }^{4}$ determinaram $\mathrm{CO}_{2}$ desprendido do solo pelos microrganismos, capturado e em solução de $\mathrm{NaOH}+\mathrm{BaCl}_{2}$ e titulado o excesso de $\mathrm{NaOH}$ com solução de $\mathrm{HCl}$. Brun e Cooper $^{5}$ avaliaram a fotossíntese das plantas utilizando o método espectrofotométrico UV pela diminuição do teor de $\mathrm{CO}_{2}$ atmosférico. Jardim et al. ${ }^{6}$ determinaram $\mathrm{CO}_{2}$ liberado em meio de cultura pela Escherichia coli em sistema de análise por injeção em fluxo (FIA) para teste antibiograma. A sensibilidade dos dois últimos métodos é na ordem de $\mathrm{mmol} \mathrm{L}^{-1}$, sendo portanto possível o seu uso para determinação do carbonato residual do calcário aplicado em solo ácido. O princípio básico do método FIA é a injeção de um volume selecionado da amostra no sistema seguido do transporte por uma solução carregadora, confluência com a solução ácida concentrada e desprendimento do $\mathrm{CO}_{2}$ da solução. $\mathrm{O}$ gás $\mathrm{CO}_{2}$ desprendido é separado através da membrana de teflon semipermeável e ao dissolver-se em água, forma os íons $\mathrm{H}^{+}$e $\mathrm{HCO}_{3}^{-}$. A concentração do $\mathrm{CO}_{2}$ é estimada pelo sinal transiente da condutância elétrica.

O objetivo do estudo foi avaliar a aplicabilidade dos sistemas de captação de $\mathrm{CO}_{2}$ e do FIA-condutometria na determinação do $\mathrm{CO}_{3}{ }^{2-}$ do solo em análises de rotina.

\section{PARTE EXPERIMENTAL}

Os componentes básicos do sistema de análise por injeção em fluxo (FIA) foram semelhantes aos utilizados por Jardim et al. ${ }^{6}$, consistindo de: uma bomba peristáltica de 4 canais, injetora de amostra, câmara de difusão de gás, célula condutivimétrica e registradora. A Figura 1 apresenta o diagrama do sistema FIA utilizado na análise. As adequações das dimensões físicas (volume da amostra injetada, vazão das solução de $\mathrm{H}_{2} \mathrm{SO}_{4}$, solução carregadora $\mathrm{KCl}$ e água destilada) e as variáveis químicas (concentração da solução de $\mathrm{H}_{2} \mathrm{SO}_{4}$ e solução carregadora $\mathrm{KCl}$ ) foram definidas em função da concentração do $\mathrm{CO}_{3}{ }^{2-}$ na amostra.

Preparou-se duas curvas analíticas de $\mathrm{CO}_{3}{ }^{2-}$, a primeira foi de 0,0 a $10,0 \mathrm{mmol} \mathrm{L}^{-1}$ e a segunda foi de 0,0 a $100,0 \mathrm{mmol}$ $\mathrm{L}^{-1}$ utilizando o reagente $\mathrm{Na}_{2} \mathrm{CO}_{3}$. A primeira curva foi para a determinação de baixa concentração de $\mathrm{CO}_{3}{ }^{2-}$ e a segunda para alta concentração, principalmente para amostras de solos que receberam de $\mathrm{CaCO}_{3}$. As vazões dos regentes do sistema FIA para a primeira curva analítica foram: $0,78 \mathrm{~mL} \mathrm{~min}^{-1}$ de $\mathrm{H}_{2} \mathrm{SO}_{4}$ $1,0 \mathrm{~mol} \mathrm{~L}^{-1} ; 0,78 \mathrm{~mL} \mathrm{~min}^{-1}$ de água destilada e $0,78 \mathrm{~mL} \mathrm{~min}^{-1}$ da solução carregadora $\mathrm{KCl} 0,5 \mathrm{~mol} \mathrm{~L}^{-1}$ e o volume da amostra injetada foi de $275 \mu \mathrm{L}$. Para alta concentração de $\mathrm{CO}_{3}{ }^{2-}$ as vazões das soluções foram de $1,2 \mathrm{~mL} \mathrm{~min}^{-1} \mathrm{de}_{2} \mathrm{SO}_{4} 1,0 \mathrm{~mol}$ $\mathrm{L}^{-1} ; 1,2 \mathrm{~mL} \mathrm{~min}^{-1}$ de água destilada e $1,2 \mathrm{~mL} \mathrm{~min}^{-1}$ da solução 


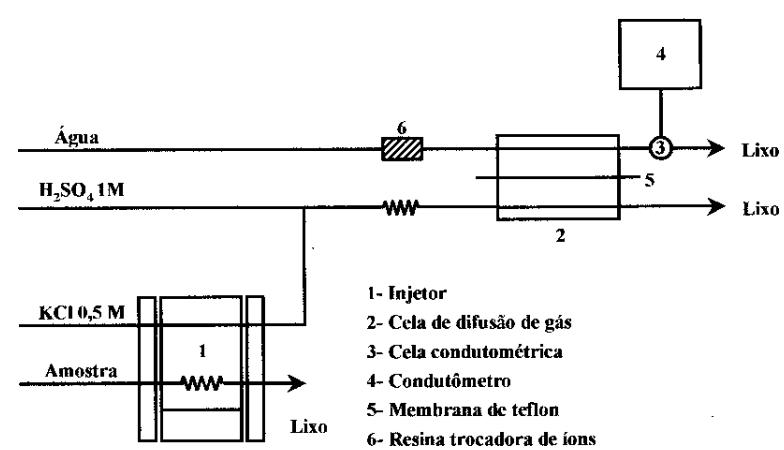

Figura 1. Diagrama do sistema FIA para determinação de $\mathrm{CO}_{3}{ }^{2-}$.

carregadora $\mathrm{KCl} 0,5 \mathrm{~mol} \mathrm{~L}^{-1}$ e o volume da amostra injetada foi de $175 \mu \mathrm{L}$. Todas as soluções foram preparadas com água destilada e fervida por 15 minutos. A solução de $\mathrm{NaOH} 0,2$ mol $\mathrm{L}^{-1}$ foi preparada a partir da solução saturada.

$\mathrm{O}$ diagrama do sistema de captação de $\mathrm{CO}_{2}$ desprendido do solo, utilizado neste experimento, está apresentado na Figura 2. O procedimento analítico foi o seguinte: pesou-se de 1,0 a 5,0 g (conforme teor de $\mathrm{CO}_{3}{ }^{2-}$ ) da amostra de solo seco, com partículas menores que $0,50 \mathrm{~mm}$, em frasco plástico de $20 \mathrm{ml}$ e em outro frasco plástico pipetou-se 2,0 ml de $\mathrm{NaOH}$ $0,2 \mathrm{~mol} \mathrm{~L}^{-1}$. Ambos os recipientes foram transferidos para frasco de vidro de $250 \mathrm{ml}$, com tampa de metal rosqueada e adicionou-se $5,0 \mathrm{ml}$ de $\mathrm{HCl} 0,5 \mathrm{~mol} \mathrm{~L}^{-1}$ na amostra de solo. $\mathrm{O}$ gás $\mathrm{CO}_{2}$ desprendido foi capturado na solução de $\mathrm{NaOH}$ e após $16 \mathrm{~h}$ determinou-se $\mathrm{CO}_{3}{ }^{2-}$ por FIA.

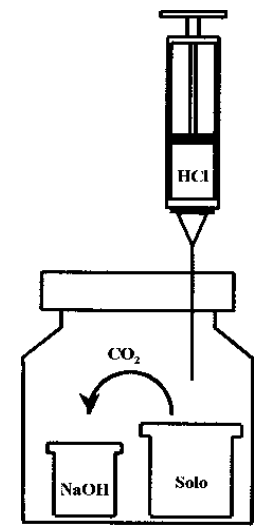

Figura 2. Sistema de captura de $\mathrm{CO}_{2}$ liberado do solo.

Para avaliar recuperação do $\mathrm{CO}_{3}{ }^{2-}$, adicionou-se 0,10 e 100 $\mathrm{mmol}_{\mathrm{c}} \mathrm{kg}^{-1}$ de $\mathrm{CaCO}_{3} \mathrm{PA}$ em amostras de solo seco passado em peneira de $0,25 \mathrm{~mm}$ e determinou-se o $\mathrm{CO}_{3}{ }^{2-}$ do solo pelo método proposto. Estas amostras também foram analisadas 10 vezes consecutivas para avaliar a precisão do método. Foram determinados o $\mathrm{CO}_{3}{ }^{2-}$ residual de 15 solos agrícolas do Paraná corrigidos com calcário comercial há mais de 8 meses e também 12 amostras de solos proveniente do controle de qualidade de análise de solos para avaliar exatidão do método.

\section{RESULTADOS E DISCUSSÃO}

As curvas analíticas do $\mathrm{CO}_{3}{ }^{2-}$ estão apresentadas nas Figuras $3 \mathrm{~A}$ e $3 \mathrm{~B}$. A não linearidade das curvas demonstram uma característica de ácido fraco $\left(\mathrm{H}_{2} \mathrm{CO}_{3}\right)$ formando-se os íons $\mathrm{H}^{+}$, $\mathrm{HCO}_{3}{ }^{-}$e $\mathrm{CO}_{3}{ }^{2-}$ na sua dissociação. A curva mostra também que a dissociação do ácido foi maior em baixas concentrações. O limite de detecção da primeira curva foi de 0,077 mmol L ${ }^{-1}$ de $\mathrm{CO}_{3}{ }^{2-}$ (Figura $3 \mathrm{~A}$ ) e da segunda curva foi de $0,83 \mathrm{mmol} \mathrm{L}^{-1}$ (Figura 3B).

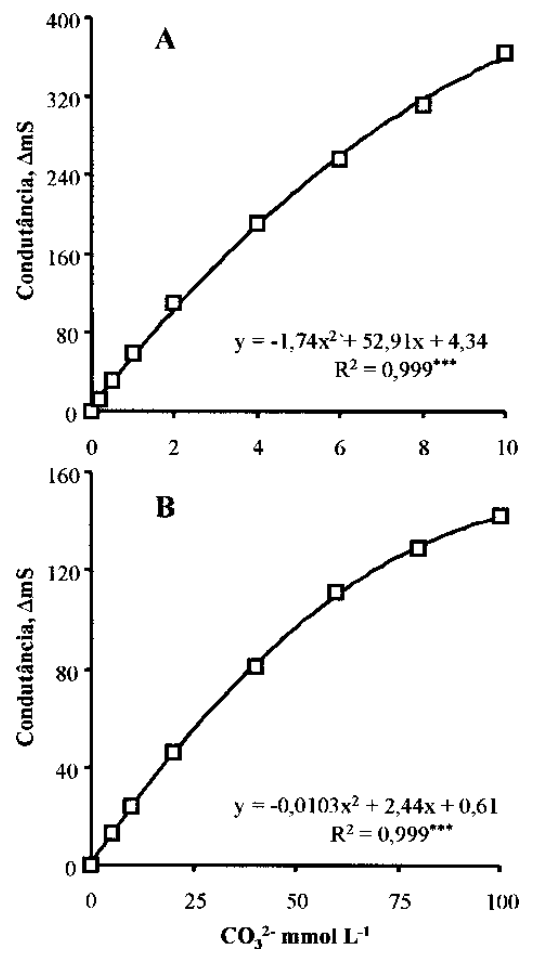

Figura 3. Curvas analíticas de $\mathrm{CO}_{3}{ }^{2-} . \mathrm{A}=0$ a $10 \mathrm{CO}_{3}{ }^{2-} \mathrm{mmol} \mathrm{L}^{-1}$ e $B=0$ a $100 \mathrm{CO}_{3}^{2-} \mathrm{mol} \mathrm{L}^{-1}$.

Avaliou-se em temperatura ambiente, o tempo necessário para captação do $\mathrm{CO}_{2}$ liberado do solo neste sistema. A fração de $\mathrm{CO}_{2}$ capturado por uma hora de um solo contendo $40 \mathrm{mmol}_{\mathrm{C}}$ $\mathrm{kg}^{-1}$ de $\mathrm{CaCO}_{3}$ foi de $61,5 \%$. Para duas e quatro horas de captação, as frações aumentaram para $71,5 \%$ e $91,7 \%$, respectivamente. A recuperação do $\mathrm{CO}_{3}{ }^{2-}$ foi completa para tempo superior a seis horas. Entretanto, o tempo de captação foi reduzido para quatro horas, quando o sistema foi aquecido a $45^{\circ} \mathrm{C}$ na estufa. Porém, como os frascos de amostras ocupam um grande volume na estufa, o processo da captação de $\mathrm{CO}_{2}$ foi feito em temperatura ambiente e o $\mathrm{CO}_{2}$ foi determinado após uma noite, aproximadamente 16 horas.

$\mathrm{O}$ sistema de captação do $\mathrm{CO}_{3}{ }^{2-}$ do solo foi avaliado adicionando-se quantidades conhecidas de $\mathrm{CaCO}_{3} \mathrm{em}$ solo ácido. $\mathrm{O}$ $\mathrm{CO}_{3}{ }^{2-}$ do solo foi determinado pelo sistema proposto e a quantidade recuperada está apresentada na Tabela 1. As quantidades entre 5 a $500 \mu \mathrm{mol}$ de $\mathrm{CO}_{3}{ }^{2-}$ do solo determinadas foram semelhantes às calculadas, apresentando uma recuperação média de $99,8 \%$. Conforme o cálculo, somente com o aumento do peso da amostra para $10 \mathrm{~g}$ pode se determinar até $0,5 \mathrm{mmol}_{\mathrm{C}}$ $\mathrm{kg}^{-1}$ de $\mathrm{CO}_{3}{ }^{2-}$ do solo. As quantidades de $\mathrm{CO}_{3}{ }^{2-}$ determinadas por 10 vezes consecutivas nestas três amostras de solos apresentaram um erro relativo médio de $2,7 \%$.

Os valores do $\mathrm{CO}_{3}{ }^{2-}$ residual determinados nas amostras de solos agrícolas corrigidos com calcário comercial e os demais parâmetros químicos: $\left(\mathrm{Ca}^{2+}, \mathrm{Mg}^{2+}, \mathrm{K}^{+}, \mathrm{Al}^{3+}\right.$ e $\left.\mathrm{pH}\right)$ de 15 amostras de solos estão apresentados na Tabela 2. Os teores de $\mathrm{CO}_{3}{ }^{2-}$ variaram de 2,6 a 134,5 $\mathrm{mmol}_{\mathrm{c}} \mathrm{kg}^{-1}$. Observou-se que para $\mathrm{pH}$ do solo inferior a 5,9, a concentração do $\mathrm{CO}_{3}{ }^{2-}$ foi menor que 4,2 $\mathrm{mmol}_{\mathrm{c}} \mathrm{kg}^{-1}$, independente dos valores de $\mathrm{Ca}, \mathrm{Mg}, \mathrm{K}$ e Al, ou seja, a concentração de $\mathrm{CO}_{3}{ }^{2-}$ em solos com $\mathrm{pH}$ inferior a 5,9 foi extremamente baixa. Quantidade apreciável de $\mathrm{CO}_{3}{ }^{2-}$ foi encontrada somente em $\mathrm{pH}$ superior a 6,1 , maior que 12,3 $\mathrm{mmol}_{\mathrm{c}} \mathrm{kg}^{-1}$. O carbonato encontrado nos solos com $\mathrm{pH}$ inferiores a 5,9, provavelmente, foram as espécies $\mathrm{HCO}_{3}{ }^{-}, \mathrm{H}_{2} \mathrm{CO}_{3} \mathrm{e}$ $\mathrm{CO}_{2}$ adsorvidos nas superfícies das partículas do solo ou grânulos de $\mathrm{CaCO}_{3}$ não reagido. Esta hipótese é provável pois o diagrama de distribuição das espécies de $\mathrm{H}_{2} \mathrm{CO}_{3} / \mathrm{CO}_{3}{ }^{2-}$ mostra 
Tabela 1. Recuperação do $\mathrm{CO}_{3}{ }^{2-}$ adicionado no solo.

\begin{tabular}{|c|c|c|c|c|c|}
\hline $\begin{array}{l}\text { peso da } \\
\text { amostra } \\
\mathrm{g}\end{array}$ & $\begin{array}{c}\mathrm{CaCO}_{3} \\
\text { adicionado } \\
\left(\mathrm{mmol}_{\mathrm{c}} \mathrm{kg}^{-1}\right)^{1}\end{array}$ & calculado & $\begin{array}{c}\mathrm{CO}_{3}{ }^{2-} \\
\text { determin. } \\
\left(\mu \mathrm{mol}_{\mathrm{c}}\right)\end{array}$ & & $(\%)$ \\
\hline 0,0 & 0,0 & - & 14,55 & - & - - \\
\hline 0,5 & 10,0 & 5,0 & 18,95 & 4,40 & 88,0 \\
\hline 1,0 & 10,0 & 10,0 & 23,90 & 9,35 & 93,5 \\
\hline 2,0 & 10,0 & 20,0 & 36,45 & 21,90 & 109,5 \\
\hline 0,5 & 100,0 & 50,0 & 63,10 & 48,55 & 97,1 \\
\hline 1,0 & 100,0 & 100,0 & 120,95 & 106,4 & 106,4 \\
\hline 2,0 & 100,0 & 200,0 & 223,90 & 209,3 & 104,6 \\
\hline 5,0 & 100,0 & 500,0 & 510,70 & 496,2 & 99,2 \\
\hline
\end{tabular}

(1) $\mathrm{mmol}_{\mathrm{c}} \mathrm{kg}^{-1}=$ miliequivalente por kg. Unidade adotada pela Sociedade Internacional da Ciência do Solo.

Tabela 2. Valores de $\mathrm{CO}_{3}{ }^{2-}$ e demais parâmetros químicos dos solos agrícolas

\begin{tabular}{|c|c|c|c|c|c|c|}
\hline $\begin{array}{c}\text { Solo } \\
n^{\circ}\end{array}$ & $\mathrm{Ca}$ & $\mathrm{Mg}$ & $\begin{array}{c}\mathrm{K} \\
\left(\mathrm{mmol}_{\mathrm{c}} \mathrm{kg}^{-1}\right)\end{array}$ & $\mathrm{Al}$ & $\mathrm{CO}_{3}{ }^{2-}$ & $\underset{\mathrm{CaCl}_{2}}{\mathrm{pH}}$ \\
\hline 1 & 23,1 & 1,8 & 0,5 & 0,5 & 4,2 & 5,0 \\
\hline 2 & 36,8 & 1,0 & 0,5 & 0,0 & 4,2 & 5,9 \\
\hline 3 & 52,2 & 0,4 & 0,5 & 0,0 & 18,0 & 7,2 \\
\hline 4 & 62,8 & 0,3 & 0,5 & 0,0 & 78,5 & 7,4 \\
\hline 5 & 64,2 & 0,2 & 0,5 & 0,0 & 99,9 & 7,5 \\
\hline 6 & 50,2 & 7,2 & 0,5 & 0,0 & 13,5 & 6,4 \\
\hline 7 & 64,8 & 5,9 & 0,7 & 0,0 & 9,9 & 6,6 \\
\hline 8 & 91,7 & 3,8 & 0,5 & 0,0 & 43,4 & 7,2 \\
\hline 9 & 110,2 & 1,4 & 0,7 & 0,0 & 88,0 & 7,6 \\
\hline 10 & 111,4 & 1,2 & 0,5 & 0,0 & 134,5 & 7,7 \\
\hline 11 & 32,8 & 3,5 & 0,7 & 1,2 & 4,2 & 4,7 \\
\hline 12 & 59,4 & 3,0 & 1,8 & 0,0 & 2,6 & 5,2 \\
\hline 13 & 88,8 & 1,7 & 1,6 & 0,0 & 12,3 & 6,1 \\
\hline 14 & 113,4 & 1,1 & 1,2 & 0,0 & 20,1 & 6,4 \\
\hline 15 & 126,2 & 0,3 & 1,6 & 0,0 & 71,5 & 7,1 \\
\hline
\end{tabular}

Tabela 3. Comparação de métodos de determinação de $\mathrm{CO}_{3}{ }^{2-}$ do solo

\begin{tabular}{|c|c|c|c|c|c|c|c|c|c|c|c|c|}
\hline & \multicolumn{12}{|c|}{$\mathrm{CO}_{3}^{2-}\left(\mathrm{mmol}_{\mathrm{c}} \mathrm{kg}^{-1}\right)$} \\
\hline ISAEP & 186 & 20 & 342 & 0 & 0 & 0 & 60 & 16 & 194 & 30 & 36 & 20 \\
\hline FIA & 166,8 & 9,4 & 355,0 & 8,2 & 7,4 & 6,8 & 58,4 & 17,4 & 177,6 & 28,4 & 38,2 & 17,8 \\
\hline
\end{tabular}

a ausência da espécie $\mathrm{CO}_{3}{ }^{2-}$ em $\mathrm{pH}$ inferior a $6,0^{7}$. Por isso, o $\mathrm{CO}_{3}{ }^{2-}$ encontrado nas amostras de solos com $\mathrm{pH}$ inferior a 6,0 seria a somatória das espécies $\mathrm{CO}_{3}{ }^{2-}, \mathrm{HCO}_{3}{ }^{-}, \mathrm{H}_{2} \mathrm{CO}_{3}$ e $\mathrm{CO}_{2}$ adsorvidas no solo.

Para avaliar a exatidão do método, foram selecionadas 12 amostras de solos com teores variando de 0 a 342 mmol $_{\mathrm{c}} \mathrm{kg}^{-1}$ de $\mathrm{CaCO}_{3}$, provenientes do controle de qualidade de análise de solos do "International Soil Analytical Exchange Programme" (ISAEP) (Tabela 3).

Os valores dos teores de $\mathrm{CaCO}_{3}$ dos solos do ISAEP e de $\mathrm{CO}_{3}{ }^{2-}$ determinados pelo método proposto estão apresentados na Tabela 3. Os dois métodos apresentaram valores similares, a equação da reta entre os métodos foi: $\mathrm{y}=0,9871 \mathrm{x}-0,075 ; \mathrm{r}^{2}$ $=0,9915$. Nas três amostras do ISAEP com teores de $0 \mathrm{mmol}_{\mathrm{c}}$ $\mathrm{kg}^{-1}$ (não detectado) foram encontrados 6,8 a 8,2 mmol $_{\mathrm{c}} \mathrm{kg}^{-1}$ de $\mathrm{CO}_{3}{ }^{2-}$ pelo método proposto. Este resultado pode ser devido a maior sensibilidade do método FIA-condutometria.

\section{CONCLUSÃO}

- $\mathrm{O}$ método proposto é aplicável na determinação de $\mathrm{CO}_{3}{ }^{2-}$ na análise de solo em rotina, com velocidade analítica de 40 amostras/ hora e com limite de detecção de 0,077 mmol $_{\mathrm{c}}$ $\mathrm{kg}^{-1}$ de $\mathrm{CO}_{3}{ }^{2-}$ do solo.

\section{REFERÊNCIAS}

1. Ziglio, C. M.; Miyazawa, M.; Pavan, M. A.; Resumo. Livro de resumos da $18^{\mathrm{a}}$ Reunião Anual da Sociedade Brasileira de Química, Caxambú, MG 1995.

2. Allison, L. E.; Moodie, C. D.; Carbonate. In: Black, C. A; Evans, D. D.; White, J. L.; Ensminger, I. E.; Clark, F. E., Eds., Method of Soil Analysis. American Society of Agronomy. Madison 1965, 9,379.

3. Ashworth, J.; Comm. Soil Sci. Plant Anal. 1997, $28,841$.

4. Jenkinson, D. S.; Powlson, D. S.; Soil Biol. Biochem. 1976, 8, 203.

5. Brun, W. A.; Cooper, R. L.; Crop Sci. 1967, 7, 451.

6. Jardim, W.; Pasquini, C.; Guimarães, J. R.; Faria, L. C.; Water Res. 1990, 24, 351.

7. Lindsay, W. L.; Chemical Equilibria in Soils; John Wiley \& Sons, New York, 1979. 